\title{
Deviation influences on sectional image reconstruction in optical scanning holography using a random-phase pupil
}

\author{
Xiu-Ling Wu, ${ }^{1}$ Xin Zhou, ${ }^{1, *}$ Qiong-Hua Wang, ${ }^{1,4}$ Yu-Fu Jiang, ${ }^{1}$ \\ Chun-Jing Xiao, ${ }^{1}$ Kelly Dobson, ${ }^{2}$ and Ting-Chung Poon ${ }^{2,3}$ \\ ${ }^{1}$ Department of Opto-Electronics Science and Technology, Sichuan University, Chengdu 610065, China \\ ${ }^{2}$ Bradley Department of Electrical and Computer Engineering, Virginia Polytechnic Institute and State University, \\ Blacksburg,Virginia 24061, USA \\ ${ }^{3}$ Shanghai Institute of Optics and Fine Mechanics, Chinese Academy of Sciences, Shanghai 201800, China \\ ${ }^{4}$ e-mail: qhwang@scu.edu.cn \\ ${ }^{*}$ Corresponding author: zhoxn@21cn.com
}

Received 8 August 2012; revised 3 October 2012; accepted 30 October 2012; posted 12 November 2012 (Doc. ID 173713); published 6 December 2012

\begin{abstract}
In this paper, we analyze the influence of two kinds of deviation errors on sectional image reconstruction for an optical scanning holography system using a random-phase pupil. The first deviation occurs in the lateral pixel position while the second occurs in the pixel value of the decoding function. Theoretical analysis and numerical simulation show that these two deviations may lead to noise in the reconstructed image. Additional discussions include the signal-to-noise ratio of the reconstructed image. (C) 2012 Optical Society of America
\end{abstract}

OCIS codes: $\quad 100.3010,280.0280,090.1995,070.2025$.

\section{Introduction}

Optical scanning holography (OSH) [1] is advantageous in real-time recording of digital holographic information due to its ability to capture a threedimensional (3-D) object with a single twodimensional (2-D) optical scan. The advantages can be similarly seen when one expands the application to 3-D optical microscopy by recording in an incoherent mode [2]. While the in-focus sectional image of 3 -D object can be reconstructed easily from the recorded electronic holograms, it is usually degraded by out-of-focus haze. The haze is a result of contributions from the other sections, which manifest as defocus noise in the in-focus section $[3,4]$. This defocus noise results from the decoding function used in the

$1559-128 \mathrm{X} / 13 / 01 \mathrm{~A} 360-07 \$ 15.00 / 0$

(C) 2013 Optical Society of America normal OSH method not being sensitive enough to the sectional location. Some efforts have been made to improve the sectional image reconstruction quality by algorithmic means [ $\underline{5}-\underline{12}]$. Wiener filtering [ $[$ ] and Wigner filtering [6] are employed in OSH reconstruction; however, they usually rely on the assumption that the object with limit sections is under ideal conditions. The norm method [7] is able to reconstruct all sectional images simultaneously while suppressing defocus noise, but it often suffers from the drawback of oversmoothing that leads the localized information to be lost. Then, edge-preserving [8] and adaptively iterative shrinkage-thresholding [9] algorithms are proposed to overcome the problem, and autofocusing [10] makes it possible to reconstruct the 3-D image of the object by focusing the images obtained from the recorded hologram using the distance parameter without any searching algorithm or tracking process. To avoid requiring a priori 
knowledge of the Fresnel zone plates, the edge detector is used to obtain an edge count function versus the reconstruction distance $[11,12]$. However, the computation of most algorithmic means is intensive.

Recently, a novel OSH method has been proposed to retrieve a section while suppressing the out-offocus haze from other sections. The principle of this approach is to use a random-phase pupil in a traditional OSH system, which structures the encoding and decoding pupil appropriately and allows noise in the reconstructed image from the defocus section to manifest as a speckle-like pattern rather than a fuzzy image [13]. Because the speckle-like pattern encompasses the entire reconstructed in-focus image, to some extent the visual effect of the in-focus image with such a speckle-like pattern is much better than that with a fuzzy haze. Additionally, this type of pattern can be easily removed by some filtering methods. The advantage of using a randomphase pupil in the OSH sectional image reconstruction is that it can effectively remove the out-of-focus sectional images without the expense of computational intensity, optimality, and estimator bias.

From this new method, matching the decoding and coding pupil functions has a great impact on the sectional image reconstruction in OSH. In this paper, we analyze the characteristics of the recovered image when the decoding pupil function results in errors either in the position of lateral direction or in the value of the pixels. Discussions also focus on the signalto-noise ratio (SNR) variation of the reconstructed sectional image.

\section{Sectional Image Reconstruction in OSH Using a Random-Phase Pupil}

An OSH system is essentially an optical image coding system for 3-D objects, as shown in Fig. 1, where lasers with frequencies of $\omega_{0}$ and $\omega_{0}+\Omega$, respectively, pass through two pupils $p_{1}(x, y)$ and $p_{2}(x, y)$ located in the front focal plane of lens $l_{1}$. The laser beams are then combined by a beam splitter (BS) and are used to $x-y$ scan the input object, which is located a distance of $z$ from the back focal plane

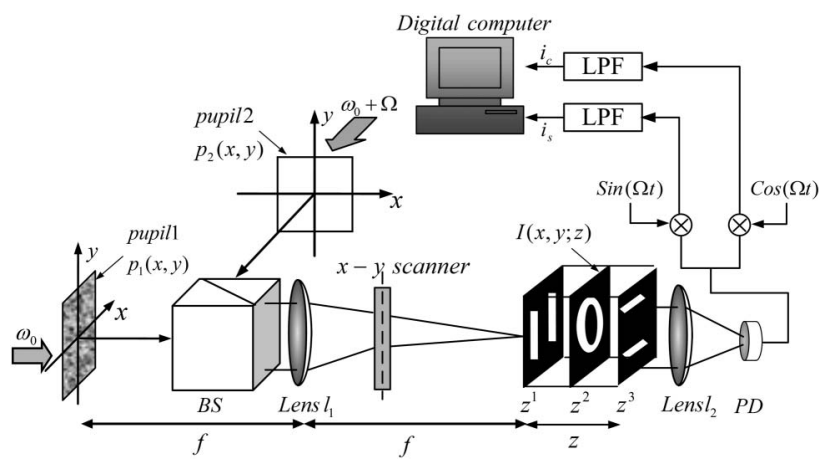

Fig. 1. Schematic of OSH system. $p_{1}(x, y)$ and $p_{2}(x, y)$ are pupil functions; $I(x, y ; z)$ is the input transmittance intensity function; $f$ is the focal length of lens $l_{1} ; z$ is the distance of the object from the focal plane of lens $l_{1} ; \otimes$ is an electronic multiplier; LPF is a low-pass filter; PD is a photo detector. of lens $l_{1}$ with a transmittance intensity function $I(x, y ; z)$. A photodetector (PD) detects the light transmitted by lens $l_{2}$, which collects all the scattered light from the object to give a heterodyne current at $\Omega$. This current is then electronically processed (by electronic multipliers and low-pass filters) as shown in Fig. 1 to give two current outputs $i_{c}(x, y)$ and $i_{s}(x, y)$. The digital computer records a complex 2-D coded hologram according to $i(x, y)=i_{c}(x, y)+j i_{s}(x, y)$ as a final output to the OSH system. Then one can reconstruct the desired digital image by deconvolving a proper decoding function with the stored coded holograms. Only if the point-spread function in the deconvolution process is close to a $2-\mathrm{D} \delta$ function can the desirable sectional image be clearly reconstructed. One can also achieve the reconstruction of a $3-\mathrm{D} \mathrm{ob-}$ ject in this way $[14,15]$.

As mentioned, the whole OSH process consists of two stages: coding and decoding. In the coding stage shown in Fig. 1 , the two pupils $p_{1}(x, y)$ and $p_{2}(x, y)$ are denoted as $p_{1 c}(x, y)$ and $p_{2 c}(x, y)$. For the decoding stage discussed later, the two pupils are denoted as $p_{1 d}(x, y)$ and $p_{2 d}(x, y)$. The output of the coding system is related to the input object $I(x, y ; z)$ located at a coding distance $z=z^{c}$ as follows [1,13]:

$$
i(x, y)=F^{-1}\left\{F\left\{I\left(x, y ; z=z^{c}\right)\right\} \cdot \mathrm{OTF}_{\Omega}\left(k_{x}, k_{y} ; z=z^{c}\right)\right\}
$$

where $\mathrm{OTF}_{\Omega}\left(k_{x}, k_{y} ; z=z^{c}\right)$ is the optical transfer function of the system given by

$$
\begin{aligned}
\mathrm{OTF}_{\Omega}\left(k_{x}, k_{y} ; z^{c}\right)= & \exp \left[j \frac{z^{c}}{2 k_{0}}\left(k_{x}^{2}+k_{y}^{2}\right)\right] \\
& \times \int p_{1 c}^{*}\left(x^{\prime}, y^{\prime}\right) p_{2 c}\left(x^{\prime}+\frac{f}{k_{0}} k_{x}, y^{\prime}+\frac{f}{k_{0}} k_{y}\right) \\
& \times \exp \left[j \frac{z^{c}}{f}\left(x^{\prime} k_{x}+y^{\prime} k_{y}\right)\right] \mathrm{d} x^{\prime} \mathrm{d} y^{\prime},
\end{aligned}
$$

where $F$ and $F^{-1}$ represent the Fourier transform and inverse Fourier transform, respectively. The superscript $*$ denotes the complex conjugate; $k_{0}$ is the wave number of the laser used; and $k_{x}$ and $k_{y}$ are the spatial frequencies.

The decoding process of the approach in [13] utilizes the same optical system as shown in Fig. 1 . with pupils $p_{1 c}(x, y)$ and $p_{2 c}(x, y)$ replaced by $p_{1 d}(x, y)$ and $p_{2 d}(x, y)$, and with a pin hole located at $z=z^{d}$ to be scanned, that is, $I\left(x, y ; z=z^{d}\right)=\delta\left(x, y ; z=z^{d}\right)$. According to Eq. (1), this gives the optical system output as $F^{-1}\left\{\mathrm{OT} \overline{\mathrm{F}}_{\Omega}\left(k_{x}, k_{y} ; z=z^{d}\right)\right\}$, representing the impulse response of the system. This is the impulse response of the optical system when $p_{1 d}(x, y)$ and $p_{2 d}(x, y)$ are used, which can be integrated with the recording process from Eq. (1) to give the final output of an overall two-stage system as [13] 


$$
\begin{aligned}
\phi_{\text {out }}(x, y)= & F^{-1}\left\{F\left\{I\left(x, y ; z^{c}\right)\right\} \cdot \mathrm{OTF}_{\Omega}\left(k_{x}, k_{y} ; z^{c}\right)\right. \\
& \left.\cdot \operatorname{OTF}_{\Omega}\left(k_{x}, k_{y} ; z^{d}\right)\right\} .
\end{aligned}
$$

In the normal OSH method [1], $p_{1 c}(x, y)=\delta(x, y)$ and $p_{2 c}(x, y)=1$ are chosen as the coding pupil function, and $p_{1 d}(x, y)=1$ and $p_{2 d}(x, y)=\delta(x, y)$ are chosen as the decoding pupil function. Substituting these functional forms of the pupils into Eq. (3) yields

$\phi_{\text {out }}(x, y)=F^{-1}\left\{F\left\{I\left(x, y ; z^{c}\right)\right\} \exp \left[j \frac{z^{d}-z^{c}}{2 k_{0}}\left(k_{x}^{2}+k_{y}^{2}\right)\right]\right\}$.

It can be seen that when $z^{d}=z^{c}$, the image at $z^{c}$ (in-focus image) can be recovered perfectly, but when $z^{d} \neq z^{c}$, the decoded image appears as a defocused image, which gives rise to noise in the form of haze in the focused image plane if the object is a 3-D image modeled as a collection of planar objects.

The method proposed in [13] uses a random-phase pupil for reconstructing the sectional image in $\mathrm{OSH}$ without the out-of-focus haze. The main difference between this approach and the normal OSH method is the use of different coding and decoding pupil functions when recording and reconstructing the sectional images. In [13] the pupil functions were chosen as $p_{1 c}(x, y)=\exp [j 2 \pi s(x, y)]$ and $p_{2 c}(x, y)=1$, where $s(x, y)$ denotes an independent random function uniformly distributed in the interval $[0,1]$. For the digital image reconstruction process the authors choose $p_{1 d}(x, y)=1$ and use phase-retrieval algorithms to adjust the decoding pupil function $p_{2 d}(x, y)$ to meet the following conditions $[\underline{13}, \underline{16}, \underline{17}]$ :

$$
\begin{gathered}
p_{1 c}^{*}(-x,-y) \cdot p_{2 d}(x, y)=1, \\
P_{1 c}^{*}\left(-\frac{z^{i}}{f} k_{x},-\frac{z^{i}}{f} k_{y}\right) \cdot P_{2 d}\left(\frac{z^{d}}{f} k_{x}, \frac{z^{d}}{f} k_{y}\right)=1,
\end{gathered}
$$

where $P_{1 c}$ and $P_{2 d}$ represent the Fourier transform of $p_{1 c}$ and $p_{2 d}$, respectively, and $z^{i}$ is the position of $i$ th planar section in a 3-D object.

Using these pupil functions in Eq. (3), the sectional image reconstruction gives [13]

$$
\begin{aligned}
\phi_{\text {out }}(x, y)= & \sum_{z^{i}} F^{-1}\left\{F\left\{I\left(x, y ; z^{i}\right)\right\} \times P_{1 c}^{*}\left(-\frac{z^{i}}{f} k_{x},-\frac{z^{i}}{f} k_{y}\right)\right. \\
& \left.\cdot P_{2 d}\left(\frac{z^{d}}{f} k_{x}, \frac{z^{d}}{f} k_{y}\right) \cdot \exp \left[j \frac{z^{d}-z^{i}}{2 k_{0}}\left(k_{x}^{2}+k_{y}^{2}\right)\right]\right\} .
\end{aligned}
$$

From Eq. (7) it can be seen that by introducing the random-phase pupil into $\mathrm{OSH}$, as long as the longitudinal position $z^{d}$ of the sectional image reconstruction is selected, the in-focus sectional image can be recovered perfectly and the defocus sectional images manifest in the form of random noise. This analysis shows that matching the decoding and coding pupil functions has a great effect on the sectional image reconstruction in OSH. However, for actual applications, the decoding and coding pupil functions usually do not exactly match because the randomphase pupil, if implemented on a spatial light modulator, becomes very sensitive to errors introduced. We shall consider two kinds of deviation errors in this paper: one in the lateral pixel position and the other in the pixel value of the decoding pupil function. Next, we will discuss effects on the reconstructed sectional image when the decoding pupil function produces both deviation errors.

\section{Deviation Influence on the Sectional Image Reconstruction}

\section{A. Lateral Deviation}

For simplicity, 1-D notation will be used in the following discussion. As shown in Fig. 2, we will denote a shift to the random-phase pupil $\bar{p}_{2 d}(x)$, for example a landscape orientation shift $x_{0}$, as $p_{2 d}^{\Delta}(x)$. Each pupil in the actual optical system has a limited size; for convenience we assume the size of the decoding random-phase mask is $D$, and the limit of pupil size in the $x$ direction is $(1 / D) \operatorname{rect}\left[\left(x-x_{0} /\right.\right.$ $\left.2) /\left(D-x_{0}\right)\right]$. We can then express $p_{2 d}^{\Delta}(x)$ as

$$
p_{2 d}^{\Delta}(x)=p_{2 d}\left(x-x_{0}\right) \cdot \frac{1}{D} \cdot \operatorname{rect}\left(\frac{x-x_{0} / 2}{D-x_{0}}\right),
$$

where $x_{0} \leq D$.

According to the shift characteristics of the Fourier transform, we can obtain following:

$$
\begin{aligned}
P_{2 d}^{\Delta}\left(\frac{z^{d}}{f} k_{x}\right)= & {\left[P_{2 d}\left(\frac{z^{d}}{f} k_{x}\right) \cdot \exp \left(-j 2 \pi \frac{z^{d}}{f} k_{x} x_{0}\right)\right] } \\
& \otimes\left[\left(\frac{D-x_{0}}{D}\right) \cdot \exp \left(-j \pi x_{0} \frac{z^{d}}{f} k_{x}\right)\right. \\
& \left.\times \sin c\left(\left(D-x_{0}\right) \frac{z^{d}}{f} k_{x}\right)\right] .
\end{aligned}
$$

The sectional image reconstruction during a shift to the random-phase pupil $p_{2 d}(x)$ from Eqs. () and (9) is then

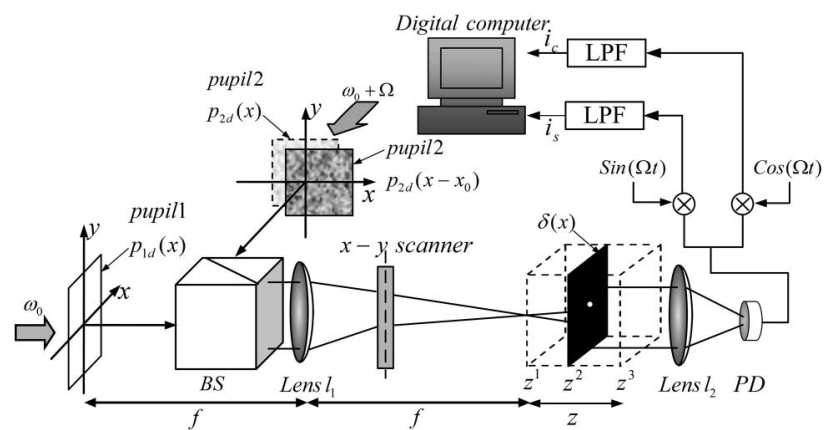

Fig. 2. Schematic of OSH system when a lateral deviation occurs. $p_{1 d}(x)$ and $p_{2 d}(x)$ are decoding pupil functions; $p_{2 d}\left(x-x_{0}\right)$ is $p_{2 d}(x)$ with a lateral shift $x_{0}$. Note that a delta function is to be scanned to obtain the point spread function of the system as discussed in the text. 


$$
\begin{aligned}
\phi_{\text {out }}(x, y)= & \sum_{z^{i}} F^{-1}\left\{F\left\{I\left(x ; z^{i}\right)\right\} P_{1 c}^{*}\left(-\frac{z^{i}}{f} k_{x}\right) P_{2 d}^{\Delta}\left(\frac{z^{d}}{f} k_{x}\right) \exp \left(j \frac{z^{d}-z^{i}}{2 k_{0}} k_{x}^{2}\right)\right\} \\
= & \sum_{z^{i}} F^{-1}\left\{F \{ I ( x ; z ^ { i } ) \} P _ { 1 c } ^ { * } ( - \frac { z ^ { i } } { f } k _ { x } ) \left\{\left[P_{2 d}\left(\frac{z^{d}}{f} k_{x}\right) \exp \left(-j 2 \pi \frac{z^{d}}{f} k_{x} x_{0}\right)\right]\right.\right. \\
& \left.\left.\otimes\left(\frac{D-x_{0}}{D}\right) \exp \left(-j \pi x_{0} \frac{z^{d}}{f} k_{x}\right) \sin c\left(\left(D-x_{0}\right) \frac{z^{d}}{f} k_{x}\right)\right\} \exp \left(j \frac{z^{d}-z^{i}}{2 k_{0}} k_{x}^{2}\right)\right\} \\
= & \sum_{z^{i}} F^{-1}\left\{F \{ I ( x ; z ^ { i } ) \} P _ { 1 c } ^ { * } ( - \frac { z ^ { i } } { f } k _ { x } ) \left\{\sum_{\xi} P_{2 d}\left(\frac{z^{d}}{f} k_{x}-\xi\right) \exp \left[-j 2 \pi\left(\frac{z^{d}}{f} k_{x}-\xi\right) x_{0}\right]\right.\right. \\
& \left.\left.\times\left(\frac{D-x_{0}}{D}\right) \exp \left(-j \pi x_{0} \xi\right) \sin c\left[\left(D-x_{0}\right) \xi\right]\right\} \exp \left(j \frac{z^{d}-z^{i}}{2 k_{0}} k_{x}^{2}\right)\right\} .
\end{aligned}
$$

The definition of convolution $f(x) \otimes g(x)=$ $\sum_{\xi} f(x-\xi) \cdot g(\xi)$ has been adopted in Eq. (10), and Eq. (10) can be expressed as

$$
\begin{aligned}
\phi_{\text {out }}(x)= & \sum_{z^{i}} F^{-1}\left\{F\left\{I\left(x ; z^{i}\right)\right\} P_{1 c}^{*}\left(-\frac{z^{i}}{f} k_{x}\right) P_{2 d}\left(\frac{z^{d}}{f} k_{x}\right)\right. \\
& \times \exp \left(-j 2 \pi \frac{z^{d}}{f} k_{x} x_{0}\right)\left(\frac{D-x_{0}}{D}\right) \\
& \left.\times \exp \left(j \frac{z^{d}-z^{i}}{2 k_{0}} k_{x}^{2}\right)+R\left(k_{x}\right)\right\},
\end{aligned}
$$

where the first term is due to $\xi=0$, the rest of the terms are grouped into $R\left(k_{x}\right)$, and its inverse Fourier transform is $r(x)$. Because $p_{1 c}(x)$ and $p_{2 d}(x)$ are random functions, $r(x)$ is also a random function. When $z^{i}=z^{d}$, and employing Eq. (6), we can obtain the reconstructed sectional image:

$$
\begin{aligned}
\phi_{\text {out }}(x)= & F^{-1}\left\{F\left\{I\left(x ; z=z^{d}\right)\right\} P_{1 c}^{*}\left(-\frac{z^{d}}{f} k_{x}\right) P_{2 d}\left(\frac{z^{d}}{f} k_{x}\right)\right. \\
& \left.\times \exp \left(-j 2 \pi \frac{z^{d}}{f} k_{x} x_{0}\right)\left(\frac{D-x_{0}}{D}\right)+R\left(k_{x}\right)\right\} \\
= & \left(\frac{D-x_{0}}{D}\right) I\left(x-\frac{z^{d}}{f} x_{0} ; z^{d}\right)+r(x) .
\end{aligned}
$$

Thus, the energy of the reconstructed sectional image can be expressed as

$$
\left|\phi_{\text {out }}(x)\right|^{2}=\left(\frac{D-x_{0}}{D}\right)^{2}\left|I\left(x-\frac{z^{d}}{f} x_{0} ; z^{d}\right)\right|^{2}+r^{\prime}(x)
$$

where $r^{\prime}(x)$ is usually a random noise, which may cause interference with the observation $[18,19]$.

From Eqs. (12) and (13), it can be seen that even for a decoding random-phase pupil lateral deviation distance $x_{0}$ the image can still be decoded, but the intensity of the reconstruction sectional image is attenuated as $x_{0}$ increases. Moreover, from Eq. (12) the reconstructed image is shifted by $z^{d} x_{0} / f$ relative to the original image.

We can evaluate the difference between the original and reconstructed images with SNR [20,21]. We normalize the energy of the original image to 1 . Because the decryption operation has been proved to be unitary [21], the energy of the reconstruction sectional image will also be 1 if the original image can be reconstructed perfectly. However, from Eq. (13) we know that the energy of the reconstruction image is $\left(D-x_{0}\right)^{2} / D^{2}$, so the remaining energy $1-$ $\left(D-x_{0}\right)^{2} / D^{2}$ must be the noise. As a result, the SNR value when the decoding pupil has a lateral direction shift can be expressed as

$$
\mathrm{SNR}=10 \log _{10}\left\{\frac{\left(D-x_{0}\right)^{2}}{D^{2}-\left(D-x_{0}\right)^{2}}\right\}
$$

As discussed above, Eq. (14) shows that as the lateral deviation of the decoding pupil increases, the energy of the reconstructed sectional image gradually attenuates and the noise energy increases. Hence, the SNR value of the reconstructed image and the intensity of the image reduce continuously.

\section{B. Pixel Value Deviation}

The next error occurs in the pixel value of the decoding random-phase pupil. Considering that any change in the distribution of the decoding random

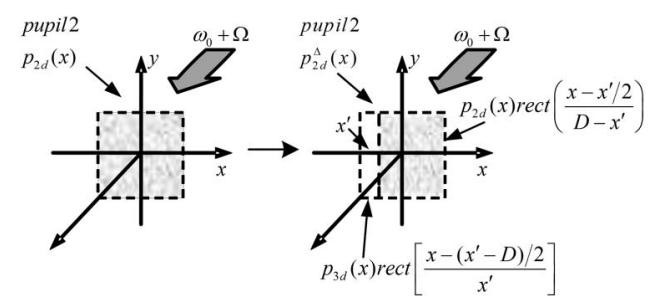

Fig. 3. Schematic of the random-phase pupil with pixel value error. 


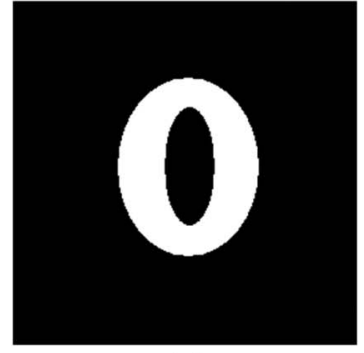

(a)

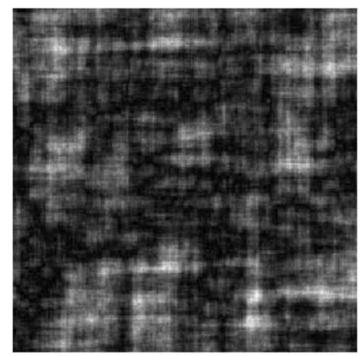

(d)

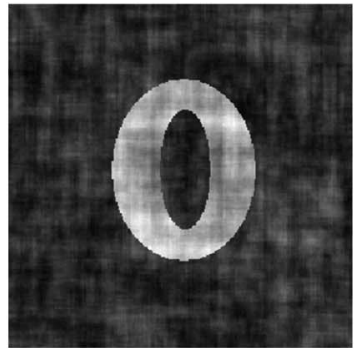

(b)

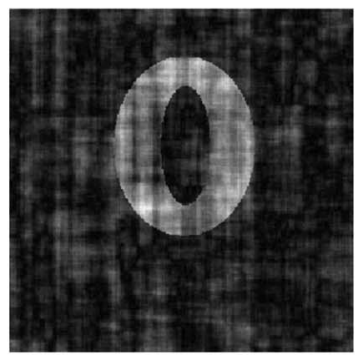

(e)

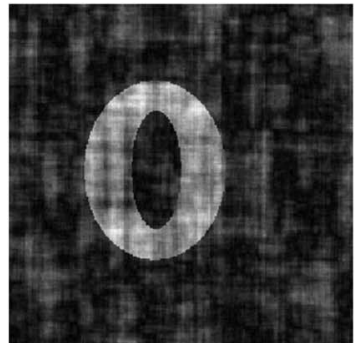

(c)

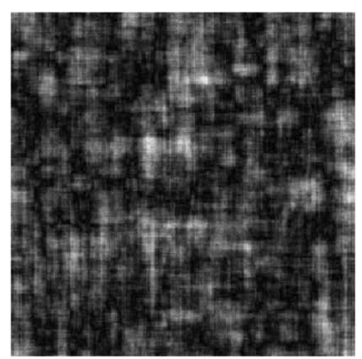

(f)

Fig. 4. Sectional image reconstruction with the decryption pupil function lateral deviation. (a) The original image; (b) $x_{0}=0 \mathrm{~mm}$, $y_{0}=0 \mathrm{~mm}$; (c) $x_{0}=2 \mathrm{~mm}, y_{0}=0 \mathrm{~mm}$; (d) $x_{0}=10 \mathrm{~mm}, y_{0}=0 \mathrm{~mm}$; (e) $x_{0}=0 \mathrm{~mm}, y_{0}=2 \mathrm{~mm}$; (f) $x_{0}=0 \mathrm{~mm}, y_{0}=10 \mathrm{~mm}$. The focus of lens $l_{1}$ is $f=7.5 \mathrm{~cm}$; the reconstruct longitudinal is $z^{d}=8 \mathrm{~cm}$.

mask, no matter where its location, results in stochastic Gaussian noise in the reconstructed image $[22,23]$, it is reasonable to assume the distribution of pixel errors in a simplistic way. Therefore, we assume that the error pixels are concentrated in a part of the random-phase pupil $p_{2 d}(x)$, and the other part remains unchanged, as shown in Fig. 3 ; that is, only a portion of the pupil function contains error pixel.

Then, the phase function of the decoding pupil can be given by

$$
\begin{aligned}
p_{2 d}^{\Delta}(x)= & \frac{1}{D}\left[p_{2 d}(x) \operatorname{rect}\left(\frac{x-x^{\prime} / 2}{D-x^{\prime}}\right)\right. \\
& \left.+p_{3 d}(x) \operatorname{rect}\left(\frac{x-\left(x^{\prime}-D\right) / 2}{x^{\prime}}\right)\right],
\end{aligned}
$$

where $p_{3 d}(x)$ is a random function and the width of value error part is $x^{\prime} \leq D$.

According to the linear superposition properties of the discrete Fourier transform, we know that

$$
\begin{aligned}
P_{2 d}^{\Delta}\left(\frac{z^{d}}{f} k_{x}\right)= & P_{2 d}\left(\frac{z^{d}}{f} k_{x}\right) \otimes\left(\frac{D-x^{\prime}}{D}\right) \exp \left(-j \pi x^{\prime} \frac{z^{d}}{f} k_{x}\right) \\
& \times \sin c\left[\left(D-x^{\prime}\right) \frac{z^{d}}{f} k_{x}\right]+P_{3 d}\left(\frac{z^{d}}{f} k_{x}\right) \\
& \otimes \frac{x^{\prime}}{D} \exp \left[-j \pi \frac{\left(x^{\prime}-D\right) z^{d}}{2 f} k_{x}\right] \sin c\left(x^{\prime} \frac{z^{d}}{f} k_{x}\right) .
\end{aligned}
$$

Using Eqs. (7), (10),(11), and (16), the reconstructed sectional image then can be obtained:

$$
\begin{aligned}
\phi_{\text {out }}(x)= & \sum_{z^{i}} F^{-1}\left\{F\left\{I\left(x ; z^{i}\right)\right\} P_{1 c}^{*}\left(-\frac{z^{i}}{f} k_{x}\right) P_{2 d}\left(\frac{z^{d}}{f} k_{x}\right)\right. \\
& \left.\times\left(\frac{D-x^{\prime}}{D}\right) \exp \left(j \frac{z^{d}-z^{i}}{2 k_{0}} k_{x}^{2}\right)+R\left(k_{x}\right)\right\} .
\end{aligned}
$$

When $z^{i}=z^{d}$ and employing Eq. ()ㅜ), Eq. (17) becomes

$$
\begin{aligned}
\phi_{\text {out }}(x)= & F^{-1}\left\{F\left\{I\left(x ; z=z^{d}\right)\right\} P_{1 c}^{*}\left(-\frac{z^{d}}{f} k_{x}\right) P_{2 d}\left(\frac{z^{d}}{f} k_{x}\right)\right. \\
& \left.\times\left(\frac{D-x^{\prime}}{D}\right)+R\left(k_{x}\right)\right\} \\
= & \left(\frac{D-x^{\prime}}{D}\right) I\left(x ; z^{d}\right)+r(x) .
\end{aligned}
$$

Note that $r(x)$ is a random noise because it rises from the mutual convolution of $I\left(x ; z^{d}\right), p_{1 c}^{*}\left(-x ; z^{d}\right)$, and $p_{3 d}\left(x ; z^{d}\right)$ as described in Eqs. (15), (16),

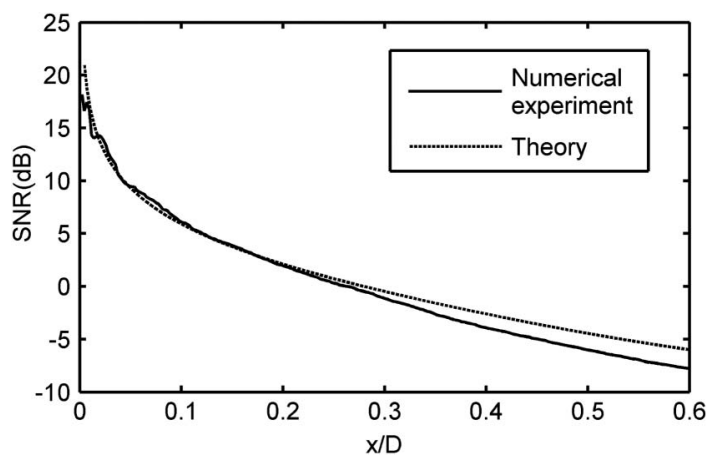

Fig. 5. Relationship curves between the normalized decoding pupil lateral shift and SNR of the reconstruction sectional image. 


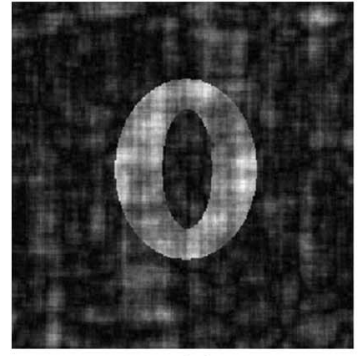

(a)

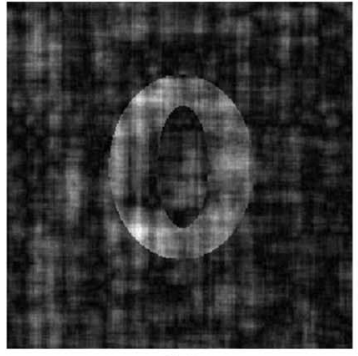

(b)

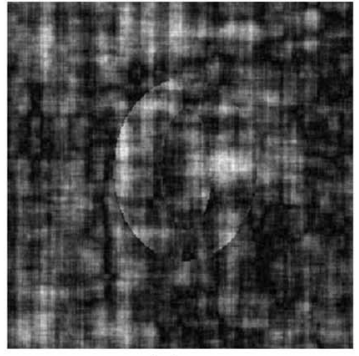

(c)

Fig. 6. Reconstructed images with error pixels of decoding pupil. (a) $1 / 196$, (b) $1 / 16$, and (c) $1 / 4$.

and (18), and obviously they are independent of each other. It should be noted that the form of Eq. (18) is almost same as that of Eq. (12), which means that the result of the reconstructed image depends mainly on the number of the value-error pixels. Therefore, as the number of error pixels increases, the noise of the reconstructed image increases and the SNR value of the reconstructed image is reduced.

\section{Computer Simulation}

\section{A. Lateral Deviation}

We have numerical simulations showing the influence of the sectional image reconstruction when the decoding random-phase pupil has a shift. The character "O", as shown in Fig. 4(a), is a binary image of $256 \times 256$ pixels and size $2 \mathrm{~cm} \times 2 \mathrm{~cm}$. In this $\mathrm{OSH}$ system, the focal length of lens $l_{1}$ is $f=7.5 \mathrm{~cm}$ and the wavelength of the laser is $\lambda=0.6328 \mu \mathrm{m}$. The sectional image at $z^{d}=8 \mathrm{~cm}$ is reconstructed in Figs. $4(\mathrm{~b})-4(\mathrm{f})$, where the decoding pupil has shifts with $x_{0}=0 \mathrm{~mm}, y_{0}=0 \mathrm{~mm} ; x_{0}=2 \mathrm{~mm}, y_{0}=0 \mathrm{~mm}$; $x_{0}=10 \mathrm{~mm}, y_{0}=0 \mathrm{~mm} ; x_{0}=0 \mathrm{~mm}, y_{0}=2 \mathrm{~mm}$; and $x_{0}=0 \mathrm{~mm}, y_{0}=10 \mathrm{~mm}$, respectively. It can be observed that when the decoding pupil function has a small lateral shift $x_{0}$ or $y_{0}$, the reconstructed sectional image also shifts in the lateral direction and additionally is quite clear. As the lateral deviation of the decoding pupil increases, the reconstructed image sharpness decreases until it can no longer be recognized.

Figure 5 presents the relationship curves between SNR of reconstructed sectional image and the

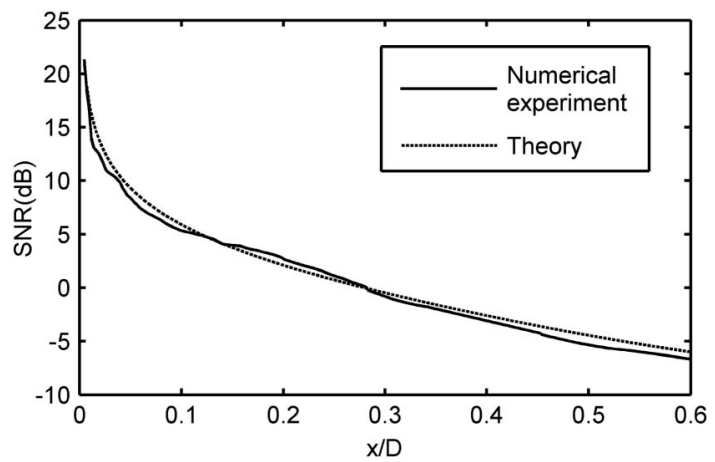

Fig. 7. Relationship curve between the ratios of the error pixels and the image SNR. normalized lateral shift $x / D$. The curve labeled "theory" in this figure is obtained from Eq. (14) and the curve labeled "numerical experiment" is from numerical simulations. These curves show that as the decoding pupil's lateral deviation increases, the noise in the reconstructed image increases, thereby eventually reducing the SNR value to the point that the sectional image reconstruction is indistinguishable.

\section{B. Pixel Value Deviation}

We use the same input image and variable parameters as presented in Fig. 4(a) for this computer simulation. Figures. 6(a)-6(c) are the reconstructed images with ratios of error pixels $1 / 196,1 / 16$, and $1 / 4$, respectively. The sectional image can be successfully reconstructed even when errors occur in the pixel value of the decoding random-phase pupil. However, as the number of error pixels gradually increases, the induced random noise increases until the reconstructed image eventually becomes unidentifiable. Figure 7 shows similar relationship curves between the ratios of error pixels and the image SNR for theory and numerical-experiment results as the lateral deviation and image SNR in Fig. 5 . Comparing these two figures, one finds that both deviations in lateral location or pixel value may lead to degradations of the reconstructed image. If implemented in a physical experiment, one should control these deviations within a range if acceptable results are desired.

\section{Conclusion}

By adopting a random-phase pupil in the $\mathrm{OSH}$ sectional image reconstruction, one can effectively reduce the defocused sectional images manifesting as haze in the in-focus image plane. In this paper, we analyze the effects of decoding-pupil-function errors in lateral shift and pixel value on sectional image reconstruction. We have presented reconstructed image results from computer simulations and discussed the SNR of these images. When a small lateral deviation occurs in the decoding pupil function, we found that the reconstructed image is also shifted and its intensity is reduced as a function of the deviation. Numerical simulations also show that when the lateral deviation becomes too large, the sectional image can no longer be distinguished. In addition, we have learned that the quality of the 
reconstructed image depends on the ratio of error pixels in the phase pupil.

This work is supported by the National Natural Science Foundation of China under Grant Nos. 61177009 and 61036008, the National "973" Program of China under Grant No. 2013CB328802, the Chinese Academy of Sciences Visiting Professorships for Senior International Scientists Program under Grant No. 2010T2G17, and the Scientific Research Foundation for the Returned Overseas Chinese Scholars, State Education Ministry.

\section{References}

1. T.-C. Poon, Optical Scanning Holography with MATLAB (Springer, 2007).

2. E. Y. Lam, X. Zhang, H. Vo, T.-C. Poon, and G. Indebetouw, "Three-dimensional microscopy and sectional image reconstruction using optical scanning holography," Appl. Opt. 48, H113-H119 (2009).

3. G. Indebetouw, "Properties of a scanning holographic microscopy: improved resolution, extended depth-of-focus, and/or optical sectioning," J. Mod. Opt. 49, 1479-1500 (2002).

4. J. Ke, T.-C. Poon, and E. Y. Lam, "Depth resolution enhancement in optical scanning holography with a dual-wavelength laser source," Appl. Opt. 50, H285-H296 (2011).

5. T. Kim, "Optical sectioning by optical scanning holography and a Wiener filter," Appl. Opt. 45, 872-879 (2006).

6. H. Kim, S.-W. Min, B. Lee, and T.-C. Poon, "Optical sectioning for optical scanning holography using phase-space filtering with Wigner distribution functions," Appl. Opt. 47, D164-D175 (2008).

7. X. Zhang, E. Y. Lam, and T.-C. Poon, "Reconstruction of sectional images in holography using inverse imaging," Opt. Express 16, 17215-17226 (2008).

8. X. Zhang and E. Y. Lam, "Edge-preserving sectional image reconstruction in optical scanning holography," J. Opt. Soc. Am. A 27, 1630-1637 (2010).

9. F.-J. Zhao, X.-C. Qu, X. Zhang, T.-C. Poon, T. Kim, Y. S. Kim, and J.-M. Liang, "Solving inverse problems for optical scanning holography using an adaptively iterative shrinkage-thresholding algorithm," Opt. Express 20, 5942-5954 (2012).

10. T. Kim and T.-C. Poon, "Autofocusing in optical scanning holography," Appl. Opt. 48, H153-H159 (2009).

11. X. Zhang, E. Y. Lam, T. Kim, Y. S. Kim, and T.-C. Poon, "Blind sectional image reconstruction for optical scanning holography," Opt. Lett. 34, 3098-3100 (2009).

12. P. W. M. Tsang, K. W. K. Cheung, T. Kim, Y. S. Kim, and T.-C. Poon, "Fast reconstruction of sectional images in digital holography," Opt. Lett. 36, 2650-2652 (2011).

13. X. Zhou, K. Dobson, Y. Shinoda, and T.-C. Poon, "Sectional image reconstruction in optical scanning holography using a random-phase pupil," Opt. Lett. 35, 2934-2936 (2010).

14. T.-C. Poon and G. Indebetouw, "Three-dimensional point spread functions of an optical heterodyne scanning image processor," Appl. Opt. 42, 1485-1492 (2003).

15. T.-C. Poon and T. Kim, "Optical image recognition of threedimensional objects," Appl. Opt. 38, 370-381 (1999).

16. J. R. Fienup, "Phase retrieval algorithms: a comparison," Appl. Opt. 21, 2758-2769 (1982).

17. R. Gerchberg and W. O. Saxton, "A practical algorithm for the determination of phase from image and diffraction plane pictures," Optik 35, 237-246 (1972).

18. B. Javidi, A. Sergent, G. Zhang, and L. Guibert, "Fault tolerance properties of a double random phase encoding encryption technique," Opt. Eng. 36, 992-998 (1997).

19. F. Goudail, F. Bollaro, B. Javidi, and P. Refregier, "Influence of a perturbation in a double phase-encoding system," J. Opt. Soc. Am. A 15, 2629-2638 (1998).

20. B. Wang, C.-C. Sun, W.-C. Su, and A. E. T. Chiou, "Shifttolerance property of an optical double-random phaseencoding encryption system," Appl. Opt. 39, 4788-4793 (2000).

21. D. Lai, X. Zhou, D.-F. Zhou, and D.-H. Li, "Study on the influence of key errors on the deciphered image in the double random phase encryption system by applying affine cryptography," J. Mod. Opt. 55, 167-176 (2008).

22. X. Zhou, S. Yuan, S. W. Wang, and J. Xie, "Affine cryptosystem of double-random-phase encryption based on the fractional Fourier transform," Appl. Opt. 45, 8434-8439 (2006).

23. T. Nomura and B. Javidi, "Optical encryption system with a binary key code,” Appl. Opt. 39, 4783-4787 (2000). 